\title{
Quenched invariance principles for random walks on percolation clusters.
}

\author{
P. Mathieu * $\quad$ A. Piatnitski ${ }^{\dagger}$
}

March 27, 2022

\begin{abstract}
We prove the almost sure ('quenched') invariance principle for a random walker on an infinite percolation cluster in $\mathbb{Z}^{d}, d \geq 2$.
\end{abstract}

\section{Introduction}

Consider super critical Bernoulli bond percolation in $\mathbb{Z}^{d}, d \geq 2$ : for $x, y \in \mathbb{Z}^{d}$, we write: $x \sim y$ if $x$ and $y$ are neighbors in the grid $\mathbb{Z}^{d}$, and let $\mathbb{E}_{d}$ be the set of non-oriented nearest pairs $(x, y)$. We identify a sub-graph of $\mathbb{Z}^{d}$ with an application $\omega \in\{0,1\}^{\mathbb{E}_{d}}$, writing $\omega(x, y)=1$ if the edge $(x, y)$ is present in $\omega$ and $\omega(x, y)=0$ otherwise. Thus $\Omega=\{0,1\}^{\mathbb{E}_{d}}$ is the set of sub-graphs of $\mathbb{Z}^{d}$. Edges pertaining to $\omega$ are then called open. Connected components of such a sub-graph will be called clusters and the cluster of $\omega$ containing a point $x \in \mathbb{Z}^{d}$ is denoted with $\mathcal{C}_{x}(\omega)$.

Define now $Q$ to be the probability measure on $\{0,1\}^{\mathbb{E}_{d}}$ under which the random variables $\left(\omega(e), e \in \mathbb{E}_{d}\right)$ are $\operatorname{Bernouilli}(p)$ independent variables and let

$$
p_{c}=\sup \left\{p ; Q\left[\# \mathcal{C}_{0}(\omega)=\infty\right]=0\right\}
$$

be the critical probability. It is known that $\left.p_{c} \in\right] 0,1[$, see [5]. Throughout the paper, we choose a parameter $p$ such that

$$
p>p_{c} .
$$

Then, $Q$ almost surely, the graph $\omega$ has a unique infinite cluster there after denoted with $\mathcal{C}(\omega)$.

We are interested in the behaviour of the simple symmetric random walk on $\mathcal{C}_{0}(\omega)$ : let $D\left(\mathbb{R}_{+}, \mathbb{Z}^{d}\right)$ be the space of càd-làg $\mathbb{Z}^{d}$-valued functions on $\mathbb{R}_{+}$and $X(t), t \in \mathbb{R}_{+}$, be the coordinate maps from $D\left(\mathbb{R}_{+}, \mathbb{Z}^{d}\right)$ to $\mathbb{Z}^{d}$. $D\left(\mathbb{R}_{+}, \mathbb{Z}^{d}\right)$ is endowed with the Skorohod topology. For a given sub-graph $\omega \in\{0,1\}^{\mathbb{E}_{d}}$, and for $x \in \mathbb{Z}^{d}$, let $P_{x}^{\omega}$ be the probability measure on $D\left(\mathbb{R}_{+}, \mathbb{Z}^{d}\right)$ under which the coordinate process is the Markov chain starting at $X(0)=x$ and with generator

$$
\mathcal{L}^{\omega} f(x)=\frac{1}{n^{\omega}(x)} \sum_{y \sim x} \omega(x, y)(f(y)-f(x)),
$$

where $n^{\omega}(x)$ is the number of neighbors of $x$ in the cluster $\mathcal{C}_{x}(\omega)$.

The behaviour of $X(t)$ under $P_{x}^{\omega}$ can be described as follows: starting from point $x$, the random walker waits for an exponential time of parameter 1 and then chooses, uniformly at random, one of its neighbors in $\mathcal{C}_{x}(\omega)$, say $y$ and moves to $y$. This procedure is then iterated with independent hoping times. The walker clearly never leaves the cluster of $\omega$ it started from. Since edges are not oriented, the measures with weights $n^{\omega}(x)$ on the possibly different clusters of $\omega$ are reversible.

\footnotetext{
*Université de Provence, CMI, 39 rue Joliot-Curie, 13013 Marseille, FRANCE. pierre.mathieu@cmi.univ-mrs.fr

${ }^{\dagger}$ Lebedev Physical Institute of Russian Academy of Sciences and Narvik Institute of Technology, P. O. Box 385, N-8505 Narvik, NORWAY. andrey@sci.lebedev.ru
} 
Let $Q_{0}$ be the conditional measure $Q_{0}()=.Q\left(. \mid \# \mathcal{C}_{0}(\omega)=\infty\right)$ and let $Q_{0} \cdot P_{x}^{\omega}$ be the so-called annealed semi-direct product measure law defined by

$$
Q_{0} \cdot P_{x}^{\omega}[F(\omega, X(.))]=\int P_{x}^{\omega}[F(\omega, X(.))] d Q_{0}(\omega) .
$$

Note that $X(t)$ is not Markovian anymore under $Q_{0} \cdot P_{x}^{\omega}$. From [3], it is known that, under $Q_{0} \cdot P_{0}^{\omega}$, the process $\left(X^{\varepsilon}(t)=\varepsilon X\left(\frac{t}{\varepsilon^{2}}\right), t \in \mathbb{R}_{+}\right)$satisfies an invariance principle as $\varepsilon$ tends to 0 i.e. it converges in law to the law of a non-degenerate Brownian motion. The proof is based on the point of view of the particule. It relies on the fact that the law of the environment $\omega$, viewed from the current position of the Markov chain is reversible, when considered under the annealed measure. It does not give any information on the behaviour of the walk for a typical choice of $\omega$. On the other hand, only partial results in dimension higher than 4 have been obtained for almost sure, also called quenched, invariance principles in the joint work of V. Sidoravicius and A-S. Sznitman, [13]. Our result holds for any dimension:

Theorem 1.1 $Q$ almost surely on the event $\# \mathcal{C}_{0}(\omega)=\infty$, under $P_{0}^{\omega}$, the process $\left(X^{\varepsilon}(t)=\varepsilon X\left(t / \varepsilon^{2}\right), t \in \mathbb{R}_{+}\right)$ converges in law as $\varepsilon$ tends to 0 to a Brownian motion with covariance matrix $\sigma^{2} I d$ where $\sigma^{2}$ is positive and does not depend on $\omega$.

Our strategy of proof follows the classical pattern introduced by S.M. Kozlov for averaging random walks with random conductances in [10]. The method of Kozlov was successfully used under ellipticity assumptions that are clearly not satisfied here. We refer in particular to first part of [13] where random walks in elliptic environments are considered. The main idea is to modify the process $X(t)$ by the addition of a corrector in such a way that the sum is a martingale under $P_{0}^{\omega}$ and use a martingale invariance principle. Then one has to prove that, in the rescaled limit, the corrector can be neglected or equivalently that the corrector has sub-linear growth. For this second step, in a classical elliptic set-up, one would invoke the Poincare inequality and the compact embedding of $H^{1}$ into $L^{2}$. For percolation models, a weaker, but still suitable form of the Poincaré inequality was proved in the paper of P. Mathieu and E. Remy [12], see also [1]. However another difficulty arises: our reference measure is the counting measure on the cluster at the origin. When rescaled, it does converge to Lebesgue measure on $\mathbb{R}^{d}$ but, for a fixed $\varepsilon$ it is of course singular. Thus rather than using classical functional analysis tools, one has to turn to $L^{2}$ techniques in varying spaces or 2 scale convergence arguments as they have been recently developped for the theory of homogenization of singular random structures in the work of A. Piatnitski and V. Jikov, see [9]. An elementary self-contained construction of the corrector is given in section 2.2. We also provide an approach to 2 scale convergence avoiding explicit reference to the results of [9]. For background material on homogenization theory in both periodic and random environments we refer to the book [8] where percolation models are considered in chapter 9 .

Note on the constants: throughout the paper $\beta$ and $c$ will denote positive constants depending only on $d$ and $p$ whose values might change from place to place.

Note: N. Berger and M. Biskup recently obtained a proof of Theorem (1.1), see [2]. Although they also rely on the construction of a corrector, their method to prove the sub-linear growth of the corrector is quite different from ours. 


\section{Proof of the theorem}

Let $|x|=\max \left|x_{i}\right|$. We use the notation $x \cdot y$ for the scalar product of the two vectors $x, y \in \mathbb{R}^{d}$. We also use the notation $Q_{0}()=.Q\left(. \mid \# \mathcal{C}_{0}(\omega)=\infty\right)$.

\subsection{Tightness}

We start recalling the Gaussian upper bound obtained by M. Barlow for walks on percolation clusters, see [1]. The corresponding lower bound also holds, but we won't need it here. Note that, Barlow's bound is only used in the proof of the tightness. Remember that $p>p_{c}$ so that, Q.a.s. the percolation sub-graph $\omega$ contains a unique infinite cluster denoted with $\mathcal{C}(\omega)$.

Statement from [1]: $Q$.a.s., for any $x \in \mathcal{C}(\omega)$ there exists a random variable $S_{x}$ such that whenever $x$ and $y$ belong to $\mathcal{C}(\omega)$ and $t \geq S_{x}$ then

$$
P_{x}^{\omega}[X(t)=y] \leq c t^{-d / 2} \exp \left(-\frac{|y-x|^{2}}{c t}\right)
$$

Moreover,

$$
Q\left[x \in \mathcal{C}(\omega), S_{x} \geq t\right] \leq c \exp \left(-c t^{\varepsilon(d)}\right) \text { with } \varepsilon(d)>0 .
$$

((2.1) is only stated with the further restriction that $t \geq|x-y|$ in [1]. If $t \leq|x-y|$, then (2.1) follows from the Carne-Varopoulos bound, see [12], appendix C.)

Lemma 2.1 $Q$ almost surely on the event $\# \mathcal{C}_{0}(\omega)=\infty$, under $P_{0}^{\omega}$, the sequence of processes $\left(X^{\varepsilon}(t)=\right.$ $\left.\varepsilon X\left(\frac{t}{\varepsilon^{2}}\right), t \in \mathbb{R}_{+}\right)$is tight in the Skorohod topology.

Proof: it is sufficient to check that $Q$.a.s. on the event $\# \mathcal{C}_{0}(\omega)=\infty$, for any $T>0$ one has

$$
\limsup _{\delta \rightarrow 0} \limsup _{\varepsilon \rightarrow 0} \sup _{\tau} E_{0}^{\omega}\left[\left|X^{\varepsilon}(\tau+\delta)-X^{\varepsilon}(\tau)\right|^{2}\right]=0,
$$

where $\tau$ is any stopping time in the filtration generated by $X^{\varepsilon}$ that is bounded by $T$. See [4], page 138 .

But, using the Markov property, we get that for large enough $K$,

$$
\begin{aligned}
& E_{0}^{\omega}\left[\left|X^{\varepsilon}(\tau+\delta)-X^{\varepsilon}(\tau)\right|^{2}\right] \\
= & \varepsilon^{2} E_{0}^{\omega}\left[\left|X\left(\frac{\tau+\delta}{\varepsilon^{2}}\right)-X\left(\frac{\tau}{\varepsilon^{2}}\right)\right|^{2}\right] \\
\leq & \varepsilon^{2} \sup _{y \in \mathcal{C}_{0}(\omega) ;|y| \leq K / \varepsilon^{2}} E_{y}^{\omega}\left[\left|X\left(\frac{\delta}{\varepsilon^{2}}\right)\right|^{2}\right]+e^{-K / 2},
\end{aligned}
$$

In the 'sup', the restriction $|y| \leq K / \varepsilon^{2}$ is justified since the walker makes more than $k$ steps in time $t$ with probability lower than $e^{-k / t}$. Since we are conditioning on the event $\mathcal{C}_{0}(\omega)=\mathcal{C}(\omega)$, one may replace the condition $y \in \mathcal{C}_{0}(\omega)$ by the condition $y \in \mathcal{C}(\omega)$ in the last term of this inequality.

From (2.1), it follows that the first term is bounded by $c \delta$ provided that $\frac{\delta}{\varepsilon^{2}} \geq \sup _{y \in \mathcal{C}(\omega) ;|y| \leq K / \varepsilon^{2}} S_{y}$. From (2.2), we get that

$$
Q\left[\sup _{y \in \mathcal{C}(\omega) ;|y| \leq K / \varepsilon^{2}} S_{y}>\frac{\delta}{\varepsilon^{2}}\right] \leq c \frac{K}{\varepsilon^{2}} \exp \left(-c\left(\frac{\delta}{\varepsilon^{2}}\right)^{\varepsilon(d)}\right) .
$$

Using the Borel-Cantelli lemma, we deduce that $Q$.a.s. on the event $\mathcal{C}_{0}(\omega)=\mathcal{C}(\omega)$

$$
\limsup _{\varepsilon \rightarrow 0} \varepsilon^{2} \sup _{y \in \mathcal{C}(\omega) ;|y| \leq K / \varepsilon^{2}} S_{y}=0
$$

and the proof is completed by letting $K$ tend to $\infty$. 


\subsection{Construction of the corrector}

Random fields: we recall that $\Omega=\{0,1\}^{\mathbb{E}_{d}}$ is the set of sub-graphs of $\mathbb{Z}^{d}$. We shall denote with $\mathcal{B}$ the set of neighbors of the origin in $\mathbb{Z}^{d}$. With some abuse of notation, we write $\omega(b)$ instead of $\omega(0, b)$ when $b \in \mathcal{B}$. We use the notation $x . \omega$ to denote the natural action of $\mathbb{Z}^{d}$ on $\Omega$ by translations. $\Omega$ is equiped with the product sigma field.

We endow $\Omega \times \mathcal{B}$ with the measure $M$ defined by

$$
\int u d M=Q\left[\sum_{b \in \mathcal{B}} \omega(b) u(\omega, b) \mathbf{1}_{\# \mathcal{C}_{0}(\omega)=\infty}\right] .
$$

Note that if two random fields $u$ and $v$ coincide in $L^{2}(\Omega \times \mathcal{B}, M)$, then, Q.a.s. on the event $\# \mathcal{C}_{0}(\omega)=\infty$, $u(\omega, b)=v(\omega, b)$ for any $b \in \mathcal{B}$ such that $\omega(b)=1$.

Let $u: \Omega \rightarrow \mathbb{R}$. $u$ is said to be local if it only depends on a finite number of coordinates. We associate to $u$ its gradient: $\nabla^{(\omega)} u: \Omega \times \mathcal{B}$ defined by

$$
\nabla^{(\omega)} u(\omega, b)=u(b . \omega)-u(\omega) .
$$

Let $L_{\text {pot }}^{2}$ be the closure in $L^{2}(\Omega \times \mathcal{B}, M)$ of the set of gradients of local fields, and $L_{\text {sol }}^{2}$ be its orthogonal complement in $L^{2}(\Omega \times \mathcal{B}, M)$.

Fields in $L_{\text {pot }}^{2}$ satisfy a co-cycle relation: on the event $\# \mathcal{C}_{0}(\omega)=\infty$, for any $u \in L_{\text {pot }}^{2}$ and any closed path in $\mathcal{C}(\omega)$ of the form $\gamma=\left(x_{0}, x_{1}, \ldots, x_{k}\right)$ with $x_{i} \sim x_{i+1}, \omega\left(x_{i}, x_{i+1}\right)=1$ and $x_{0}=x_{k}=0$ then $\sum_{i=1}^{k} u\left(x_{i-1} \cdot \omega, x_{i}-\right.$ $\left.x_{i-1}\right)=0$.

Let us write down explicitely what it means for a square integrable field $v$ to be in $L_{\text {sol }}^{2}$ : let $u$ be a local function on $\Omega$. Then

$$
\begin{aligned}
& Q\left[\left(\sum_{b \in \mathcal{B}} \omega(b) v(\omega, b) \nabla^{(\omega)} u(\omega, b) \mathbf{1}_{\# \mathcal{C}_{0}(\omega)=\infty}\right]=Q\left[\sum_{b \in \mathcal{B}} v(\omega, b) \nabla^{(\omega)} u(\omega, b) \mathbf{1}_{0 \in \mathcal{C}(\omega), b \in \mathcal{C}(\omega)]}\right]\right. \\
= & Q\left[\sum_{b \in \mathcal{B}} v(\omega, b)(u(b . \omega)-u(\omega)) \mathbf{1}_{0 \in \mathcal{C}(\omega), b \in \mathcal{C}(\omega)] .}\right.
\end{aligned}
$$

Using the translation invariance of $Q$, we then get that

$$
\begin{aligned}
& Q\left[\sum_{b \in \mathcal{B}} v(\omega, b) u(b . \omega) \mathbf{1}_{0 \in \mathcal{C}(\omega), b \in \mathcal{C}(\omega)}\right]=Q\left[\sum_{b \in \mathcal{B}} v((-b) . b . \omega, b) u(b . \omega) \mathbf{1}_{0 \in \mathcal{C}(b . \omega),-b \in \mathcal{C}(b . \omega)}\right] \\
& =Q\left[\sum_{b \in \mathcal{B}} v((-b) . \omega, b) u(\omega) \mathbf{1}_{0 \in \mathcal{C}(\omega),-b \in \mathcal{C}(\omega)}\right]=Q\left[\sum_{b \in \mathcal{B}} v(b . \omega,-b) u(\omega) \mathbf{1}_{0 \in \mathcal{C}(\omega), b \in \mathcal{C}(\omega)}\right] \\
& =Q\left[\sum_{b \in \mathcal{B}} \omega(b) v(b . \omega,-b) u(\omega) \mathbf{1}_{\# \mathcal{C}_{0}(\omega)=\infty}\right] .
\end{aligned}
$$

So that

$$
Q\left[\sum_{b \in \mathcal{B}} v(\omega, b)(u(b . \omega)-u(\omega)) \mathbf{1}_{0 \in \mathcal{C}(\omega), b \in \mathcal{C}(\omega)]}=Q\left[\sum_{b \in \mathcal{B}} \omega(b) u(\omega)(v(b . \omega,-b)-v(\omega, b)) \mathbf{1}_{\# \mathcal{C}_{0}(\omega)=\infty}\right] .\right.
$$

Thus we have proved the following integration by parts formula:

$$
\int v \nabla^{(\omega)} u d M=-Q\left[n^{\omega}(0) u \nabla^{(\omega) *} v \mathbf{1}_{\# \mathcal{C}_{0}(\omega)=\infty}\right]
$$

where

$$
\nabla^{(\omega) *} v(\omega)=\frac{1}{n^{\omega}(0)} \sum_{b \in \mathcal{B}} \omega(b)(v(\omega, b)-v(b . \omega,-b))
$$

(2.3) holds for a square integrable random field $v$ and any local function $u$. 
As a consequence, taking $v$ to be constant, note that $\int \nabla^{(\omega)} u d M=0$ for any local $u$. By extension, we will also have $\int u d M=0$ for any $u \in L_{\text {pot }}^{2}$.

A square integrable random field $v$ is in $L_{\text {sol }}^{2}$ if it satisfies $\nabla^{(\omega) *} v=0$ Q.a.s. on the set $\# \mathcal{C}_{0}(\omega)=\infty$.

Definition of the corrector: let $b \in \mathcal{B}$. Define the random field $\hat{b}(\omega, e)=\mathbf{1}_{e=b}-\mathbf{1}_{e=-b}$. Let $G_{b}$ be the unique solution in $L_{\text {pot }}^{2}$ satisfying the equation

$$
\hat{b}+G_{b}(\omega, e) \in L_{s o l}^{2} .
$$

$\left(G_{b}\right.$ is simply the projection of $-\hat{b}$ on $L_{\text {pot }}^{2}$.)

We define the corrector $\chi: \Omega \times \mathcal{C}_{0}(\omega) \rightarrow \mathbb{R}^{d}$ by the equation

$$
\chi(\omega, x+e) \cdot b-\chi(\omega, x) \cdot b=G_{b}(x . \omega, e),
$$

for any $x \in \mathbb{Z}^{d}, b, e \in \mathcal{B}$. (In this equation $\chi(.) \cdot b$ stands for the usual scalar product of the two $\mathbb{R}^{d}$ vectors $\chi($.$) and b$. Note that there is no ambiguity because $G_{b}=-G_{-b}$ as can be directly seen from equation (2.4).) Observe that, unlike $G_{b}$, the corrector $\chi$ is not an homogeneous field.

The solution to (2.4) being unique in $L_{\text {pot }}^{2}$, the value of $G_{b}(\omega, e)$ is uniquely determined whenever $\# \mathcal{C}_{0}(\omega)=\infty$ and $e \in \mathcal{B}$ satisfies $\omega(e)=1$. Therefore $G_{b}(x . \omega, e)$ is well defined $Q$.a.s. on the set $\# \mathcal{C}_{0}(\omega)=\infty$ for any $x$ and $e$ s.t. $x$ and $x+e$ belong to $\mathcal{C}(\omega)$. Thus, if $x$ belongs to $\mathcal{C}_{0}(\omega)$, then the value of $\chi(\omega, x)-\chi(\omega, 0)$ can be computed integrating (2.5) along a path in $\mathcal{C}_{0}(\omega)$ from the origin to $x$. That this value does not depend on the choice of the path is an immediate consequence of the co-cycle relation satisfied by $G_{b}$. We conclude that $\chi(\omega, x)$ is uniquely determined by equation (2.5) up to an additive constant (that might depend on $\omega$ ).

The martingale property: we claim that the random process $X(t)+\chi(\omega, X(t))$ is a martingale under $P_{0}^{\omega}$ for $Q$ almost all $\omega$ s.t. $\# \mathcal{C}_{0}(\omega)=\infty$. Note that since the process $X(t)$, starting from the origin, never leaves $\mathcal{C}(\omega), \chi(\omega, X(t))$ is indeed well defined.

We choose $\omega$ s.t. $\# \mathcal{C}_{0}(\omega)=\infty$.

Since $G_{b} \in L_{\text {pot }}^{2}$, the co-cycle relation implies that $G_{b}(\omega, e)+G_{b}(e \cdot \omega,-e)=0$ for any $e \in \mathcal{B}$ s.t. $\omega(e)=1$. Comparing the expression of $\mathcal{L}^{\omega}$ with the definition of $\nabla^{(\omega) *}$, we then see that $\mathcal{L}^{\omega} \chi(\omega, x) \cdot b=\frac{1}{2} \nabla^{(\omega) *} G_{b}(x . \omega)$ for any $x \in \mathcal{C}(\omega)$.

Let $\phi(x)=x+\chi(\omega, x)$. Noting that $\hat{b}(\omega, e)=e \cdot b$ and that $\hat{b}(e \cdot \omega,-e)=-b \cdot e$, we see that $\nabla^{(\omega) *} \hat{b}(\omega)=$ $\frac{2}{n^{\omega}(0)} \sum_{e \in \mathcal{B}} \omega(e) b \cdot e$. Therefore

$$
\begin{aligned}
& \mathcal{L}^{\omega} \phi(\omega, x) \cdot b=\frac{1}{n^{\omega}(x)} \sum_{e \in \mathcal{B}} \omega(x, x+e) e \cdot b+\mathcal{L}^{\omega} \chi(\omega, x) \\
= & \frac{1}{2} \nabla^{(\omega) *} \hat{b}(x \cdot \omega)+\frac{1}{2} \nabla^{(\omega) *} G_{b}(x \cdot \omega)=0 .
\end{aligned}
$$

This last equality holds for any $x \in \mathcal{C}(\omega)$. We have proved the martingale property.

The invariance principle: to each pair of neighbouring points $x, y \in \mathcal{C}_{0}(\omega)$ such that $\omega(x, y)=1$ attach a Poisson process of rate $1 / n^{\omega}(x)$, say $N_{t}^{x, y}$, all of them independent. Let $Y$ be the solution of the equation $Y(0)=0$,

$$
d Y(t)=\sum_{y \sim Y(t-)} \omega(Y(t-), y)(y-Y(t-)) d N_{t}^{Y(t-), y} .
$$

Then the law of the random process $(Y(t), t \geq 0)$ is $P_{0}^{\omega}$.

Let $\omega$ be such that $\# \mathcal{C}_{0}(\omega)=\infty$. Let $N(t)=Y(t)+\chi(\omega, Y(t))$. From the previous paragraph, we already know that $N$ is a martingale. Its bracket can be computed using Itô's formula. We fix a direction $b \in \mathcal{B}$. Then:

$$
\begin{aligned}
& d<N \cdot b>(t) \\
= & \frac{1}{n^{\omega}(Y(t-))} \sum_{y \sim Y(t-)} \omega(Y(t-), Y(t-)+e)(y \cdot b+\chi(\omega, y) \cdot b-Y(t-) \cdot b-\chi(\omega, Y(t-)) \cdot b)^{2} d t
\end{aligned}
$$




$$
=\frac{1}{n^{Y(t-) \cdot \omega}(0)} \sum_{e \in \mathcal{B}} Y(t-) \cdot \omega(e)\left(e \cdot b+G_{b}(Y(t-) \cdot \omega, e)\right)^{2} d t .
$$

Back to the process $X$ and denoting $M(t)=X(t)+\chi(\omega, X(t))$, we can equivalently write that

$$
d<M \cdot b>(t)=\frac{1}{n^{X(t-) \cdot \omega}(0)} \sum_{e \in \mathcal{B}} X(t-) \cdot \omega(e)\left(e \cdot b+G_{b}(X(t-) \cdot \omega, e)\right)^{2} d t .
$$

Let $\tilde{Q}_{0}$ be the probability measure

$$
\tilde{Q}_{0}(A)=\frac{\int_{A} n^{\omega}(0) d Q_{0}(\omega)}{\int n^{\omega}(0) d Q_{0}(\omega)}
$$

$X(t-) . \omega$ is the process of the environment viewed from the particule. The measure $\tilde{Q}_{0}$ is reversible, invariant and ergodic with respect to $X(t-) . \omega$, see Lemma 4.9 in [3]. As a consequence, we get that, Q.a.s. on the set $\# \mathcal{C}_{0}(\omega)=\infty$

$$
\frac{\leq M \cdot b>(t)}{t} \rightarrow \tilde{Q}_{0}\left(\frac{1}{n^{\omega}(0)} \sum_{e \in \mathcal{B}} \omega(e)\left(e \cdot b+G_{b}(\omega, e)\right)^{2}\right) .
$$

Let now $M^{\varepsilon}(t)=\varepsilon M\left(t / \varepsilon^{2}\right)$. We have proved that, for any $t>0$, as $\varepsilon$ tends to 0

$$
<M^{\varepsilon} \cdot b>(t) \rightarrow t \tilde{Q}_{0}\left(\frac{1}{n^{\omega}(0)} \sum_{e \in \mathcal{B}} \omega(e)\left(e \cdot b+G_{b}(\omega, e)\right)^{2}\right) .
$$

For any function $f$ that vanishes on the diagonal, the process

$$
\sum_{0 \leq s \leq t} f(X(s), X(s-))-\int_{0}^{t} d s \frac{1}{n^{X(s-) \cdot \omega}(0)} \sum_{e \in \mathcal{B}} X(s-) \cdot \omega(e) f(X(s-)+e, X(s-))
$$

is a martingale. Applying this to $f(x, y)=(b \cdot(x+\chi(\omega, x))-b \cdot(y+\chi(\omega, y)))^{2} \mathbf{1}_{|b \cdot(x+\chi(\omega, x))-b \cdot(y+\chi(\omega, y))| \geq \eta}$ for some direction $b$ and some $\eta>0$, we get that

$$
\begin{aligned}
\sum_{0 \leq s \leq t} & (M(s) \cdot b-M(s-) \cdot b)^{2} \mathbf{1}_{|M(s) \cdot b-M(s-) \cdot b| \geq \eta} \\
- & \int_{0}^{t} d s \frac{1}{n^{X(s-) \cdot \omega}(0)} \sum_{e \in \mathcal{B}} X(s-) \cdot \omega(e)\left(e \cdot b+G_{b}(X(s-) \cdot \omega, e)\right)^{2} \mathbf{1}_{\left|e \cdot b+G_{b}(X(s-) \cdot \omega, e)\right| \geq \eta}
\end{aligned}
$$

is a martingale. Taking expectations and using the ergodic theorem for the process $X(s-) . \omega$ we get that, on the set $\# \mathcal{C}_{0}(\omega)=\infty$,

$$
\begin{aligned}
& E_{0}^{\omega}\left[\frac{1}{t} \sum_{0 \leq s \leq t}(M(s) \cdot b-M(s-) \cdot b)^{2} \mathbf{1}_{|M(s) \cdot b-M(s-) \cdot b| \geq \eta}\right] \\
= & \frac{1}{t} \int_{0}^{t} d s E_{0}^{\omega}\left[\frac{1}{n^{X(s-) \cdot \omega}(0)} \sum_{e \in \mathcal{B}} X(s-) \cdot \omega(e)\left(e \cdot b+G_{b}(X(s-) \cdot \omega, e)\right)^{2} \mathbf{1}_{\left|e \cdot b+G_{b}(X(s-) \cdot \omega, e)\right| \geq \eta}\right] \\
\rightarrow & \tilde{Q}_{0}\left(\frac{1}{n^{\omega}(0)} \sum_{e \in \mathcal{B}} \omega(e)\left(e \cdot b+G_{b}(\omega, e)\right)^{2} \mathbf{1}_{\left|e \cdot b+G_{b}(\omega, e)\right| \geq \eta}\right)<\infty .
\end{aligned}
$$

Then, for any $t>0$

$$
\begin{aligned}
& E_{0}^{\omega}\left[\sum_{0 \leq s \leq t}\left(M^{\varepsilon}(s) \cdot b-M^{\varepsilon}(s-) \cdot b\right)^{2} \mathbf{1}_{\left|M^{\varepsilon}(s) \cdot b-M^{\varepsilon}(s-) \cdot b\right| \geq \eta}\right] \\
= & \varepsilon^{2} E_{0}^{\omega}\left[\sum_{0 \leq s \leq t / \varepsilon^{2}}(M(s) \cdot b-M(s-) \cdot b)^{2} \mathbf{1}_{|M(s) \cdot b-M(s-) \cdot b| \geq \eta / \varepsilon}\right]
\end{aligned}
$$


$\rightarrow 0$.

From the martingale convergence theorem, Theorem 5.1 part a in [7], we then deduce that, Q.a.s. on the set $\# \mathcal{C}_{0}(\omega)=\infty$, the law of the process $\varepsilon X\left(. / \varepsilon^{2}\right)+\varepsilon \chi\left(\omega, X\left(. / \varepsilon^{2}\right)\right)$ under $P_{0}^{\omega}$ converges to the law of a Brownian motion with a deterministic covariance matrix $A$.

That $A$ is diagonal is proved in [3], Theorem 4.7,3. One can argue that $A$ is positive as a consequence of the Gaussian lower bounds obtained in [1], but the original proof is given in [6].

We therefore conclude that Theorem 1.1 will follow if we can prove that, Q.a.s. on the set $\# \mathcal{C}_{0}(\omega)=\infty$, for all $t>0, \varepsilon \chi\left(\omega, X\left(t / \varepsilon^{2}\right)\right)$ converges to 0 in $P_{0}^{\omega}$ probability.

\subsection{Convergence of the corrector}

We now check that the contribution of the corrector is negligible in the limit i.e. we prove that, for all $t$, $\varepsilon \chi\left(\omega, X\left(t / \varepsilon^{2}\right)\right)$ converges to 0 in $P_{0}^{\omega}$ probability, $Q$.a.s. on the set $\# \mathcal{C}_{0}(\omega)=\infty$. In view of $(2.1)$, it is sufficient to show that

$$
\lim _{\varepsilon \rightarrow 0} \varepsilon^{d} \sum_{y \in \mathcal{C}_{0}(\omega) ;|y| \leq 1 / \varepsilon}|\varepsilon \chi(\omega, y)|^{2}=0 \quad Q_{0} \text {.a.s. . }
$$

Below, we use the Poincaré inequality to prove that there exist some constants $a_{\varepsilon}(\omega)$ such that

$$
\lim _{\varepsilon \rightarrow 0} \varepsilon^{d} \sum_{y \in \mathcal{C}_{0}(\omega) ;|y| \leq 1 / \varepsilon}\left|\varepsilon \chi(\omega, y)-a_{\varepsilon}\right|^{2}=0 \quad Q_{0} . \text { a.s. . }
$$

As a consequence of (2.1), (2.6) implies that

$$
\lim _{t \rightarrow 0} \lim _{\varepsilon \rightarrow 0} P_{0}^{\omega}\left[\left|\varepsilon \chi\left(\omega, X\left(\frac{t}{\varepsilon^{2}}\right)\right)-a_{\varepsilon}\right| \geq K\right]=0 \quad Q_{0} \text {.a.s., and for any } K>0 .
$$

But the invariance principle for the process $\varepsilon X\left(\frac{t}{\varepsilon^{2}}\right)+\varepsilon \chi\left(\omega, X\left(\frac{t}{\varepsilon^{2}}\right)\right)$ implies that

$$
\lim _{t \rightarrow 0} \lim _{\varepsilon \rightarrow 0} P_{0}^{\omega}\left[\left|\varepsilon X\left(\frac{t}{\varepsilon^{2}}\right)+\varepsilon \chi\left(\omega, X\left(\frac{t}{\varepsilon^{2}}\right)\right)\right| \geq K\right]=0 \quad Q_{0} \text {.a.s., and for any } K>0,
$$

and (2.1) implies that

$$
\lim _{t \rightarrow 0} \lim _{\varepsilon \rightarrow 0} P_{0}^{\omega}\left[\left|\varepsilon X\left(\frac{t}{\varepsilon^{2}}\right)\right| \geq K\right]=0 \quad Q_{0} \text {.a.s., and for any } K>0 .
$$

Therefore

$$
\lim _{t \rightarrow 0} \lim _{\varepsilon \rightarrow 0} P_{0}^{\omega}\left[\left|\varepsilon \chi\left(\omega, X\left(\frac{t}{\varepsilon^{2}}\right)\right)\right| \geq K\right]=0 \quad Q_{0} \text {.a.s., and for any } K>0 .
$$

Thus we see that $a_{\varepsilon}$ tends to 0 and

$$
\lim _{\varepsilon \rightarrow 0} \varepsilon^{d} \sum_{y \in \mathcal{C}_{0}(\omega) ;|y| \leq 1 / \varepsilon}|\varepsilon \chi(\omega, y)|^{2}=0 \quad Q_{0} . \text { a.s. . }
$$

It remains to justify (2.6).

Poincaré inequalities: since $G_{b}$ is square integrable, the spatial ergodic theorem, see [11] page 205, implies that $\varepsilon^{d} \sum_{e \in \mathcal{B}} \sum_{x \in \mathcal{C}_{0}(\omega) ;|x| \leq 1 / \varepsilon} \omega(e)\left(G_{b}(x . \omega, e)\right)^{2}$ has a Q.a.s. finite limit. Therefore

$$
\limsup _{\varepsilon} \varepsilon^{d} \sum_{e \in \mathcal{B}} \sum_{x \in \mathcal{C}_{0}(\omega) ;|x| \leq 1 /(1-a) \varepsilon} x . \omega(e)\left(G_{b}(x . \omega, e)\right)^{2}<\infty,
$$

$Q_{0}$.a.s. and for any constant $0<a<1$. 
We quote from [12], Theorem 1.3: for some $\varepsilon>0$ define $\mathcal{C}^{\varepsilon}$ to be the connected component of the intersection of $\mathcal{C}_{0}(\omega)$ with the box $\left[-\frac{1}{\varepsilon}, \frac{1}{\varepsilon}\right]^{d}$ that contains the origin. There exists a constant $\beta$ such that $Q_{0}$.a.s. for small enough $\varepsilon$, for any function $u: \mathcal{C}^{\varepsilon} \rightarrow \mathbb{R}$ one has

$$
\frac{1}{\# \mathcal{C}^{\varepsilon}} \sum_{x, y \in \mathcal{C}^{\varepsilon}}(u(x)-u(y))^{2} \leq \beta \varepsilon^{-2} \sum_{x \sim y \in \mathcal{C}^{\varepsilon}} \omega(x, y)(u(x)-u(y))^{2} .
$$

Since $\# \mathcal{C}^{\varepsilon}$ is of order $\varepsilon^{-d}$ for small enough $\varepsilon$ and since $\mathcal{C}_{0}(\omega) \cap\left[-\frac{1}{\varepsilon}, \frac{1}{\varepsilon}\right]^{d} \subset \mathcal{C}^{(1-a) \varepsilon}$ for some constant $a$, we therefore have a constant $\beta$ such that, $Q_{0}$.a.s. for small enough $\varepsilon$, for any function $u: \mathcal{C}(\omega) \rightarrow \mathbb{R}$

$$
\varepsilon^{d} \sum_{x, y \in \mathcal{C}(\omega) ;|x|,|y| \leq 1 / \varepsilon}(u(x)-u(y))^{2} \leq \beta \varepsilon^{-2} \sum_{x \sim y \in \mathcal{C}^{(1-a) \varepsilon}} \omega(x, y)(u(x)-u(y))^{2} .
$$

We use this last inequality for the function $u(x)=\chi(\omega, x)$ to get that,

$$
\varepsilon^{d} \sum_{x, y \in \mathcal{C}(\omega) ;|x|,|y| \leq 1 / \varepsilon}(\chi(\omega, x)-\chi(\omega, y))^{2} \leq \beta \varepsilon^{-2} \sum_{b \in \mathcal{B}} \sum_{e \in \mathcal{B}} \sum_{x \in \mathcal{C}_{0}(\omega) ;|x| \leq 1 /(1-a) \varepsilon} x . \omega(e)\left(G_{b}(x . \omega, e)\right)^{2} .
$$

By (2.7) we therefore get that

$$
\limsup _{\varepsilon} \varepsilon^{2 d} \sum_{x, y \in \mathcal{C}(\omega) ;|x|,|y| \leq 1 / \varepsilon}(\varepsilon \chi(\omega, x)-\varepsilon \chi(\omega, y))^{2}<\infty
$$

$Q_{0}$. a.s., and thus

$$
\limsup _{\varepsilon} \varepsilon^{d} \sum_{x \in \mathcal{C}(\omega) ;|x| \leq 1 / \varepsilon}\left(\varepsilon \chi(\omega, x)-a_{\varepsilon}\right)^{2}<\infty,
$$

$Q_{0}$.a.s. where $a_{\varepsilon}$ is the mean value of $\varepsilon \chi(\omega, x)$ on the set $\{x \in \mathcal{C}(\omega) ;|x| \leq 1 / \varepsilon\}$.

Two scale convergence: we first introduce some notation. Let $G=]-1,1\left[{ }^{d}\right.$. For $\omega \in \Omega$ and $\varepsilon>0$, we define the measures

$$
\mu_{\omega}=\sum_{z \in \mathcal{C}(\omega)} n^{\omega}(z) \delta_{z}, \mu_{\omega}^{\varepsilon}=\varepsilon^{d} \sum_{z \in \mathcal{C}(\omega)} n^{\omega}(z) \delta_{\varepsilon z}
$$

Given a direction $e \in \mathcal{B}$, the gradient of a function $\phi: \mathbb{R}^{d} \rightarrow \mathbb{R}$ is

$$
\nabla_{e}^{\varepsilon} \phi(z)=\frac{1}{\varepsilon}(\phi(z+\varepsilon e)-\phi(z)) .
$$

Let us now choose $b_{0} \in \mathcal{B}$ and let

$$
\psi^{\varepsilon}(\omega, z)=\left(\varepsilon \chi\left(\omega, \frac{1}{\varepsilon} z\right)-a_{\varepsilon}\right) \cdot b_{0} .
$$

Thus $\psi^{\varepsilon}$ is well defined for $z \in \varepsilon \mathcal{C}_{0}(\omega)$. From the definition of $\chi$, we have

$$
\nabla_{e}^{\varepsilon} \psi^{\varepsilon}(\omega, z)=G_{b_{0}}\left(\frac{1}{\varepsilon} z \cdot \omega, e\right)
$$

for $z \in \varepsilon \mathcal{C}_{0}(\omega)$.

Keep in mind that for $z^{\prime} \in \mathbb{Z}^{d}$, the expression $z^{\prime} . \omega$ denotes the graph obtained by translating $\omega$ by $z^{\prime}$. In particular, for $z \in \varepsilon \mathbb{Z}^{d}$, then $\frac{1}{\varepsilon} z . \omega(e)$ is either 0 or 1 , depending on wether the edge $(z, z+e)$ belongs to $\omega$ or not. We sometimes prefer the notation $\left(\frac{z}{\varepsilon}\right) . \omega(e)$ in order to avoid possible confusion.

In our new notation, (2.7) and (2.8) now read:

$$
C_{1}(\omega)=\sup _{e \in \mathcal{B}} \sup _{\varepsilon} \int_{G}\left(\frac{z}{\varepsilon}\right) \cdot \omega(e)\left(\nabla_{e}^{\varepsilon} \psi^{\varepsilon}(\omega, z)\right)^{2} d \mu_{\omega}^{\varepsilon}(z)<\infty,
$$

and

$$
C_{2}(\omega)=\sup _{\varepsilon} \int_{G}\left(\psi^{\varepsilon}(\omega, z)\right)^{2} d \mu_{\omega}^{\varepsilon}(z)<\infty
$$


for $Q_{0}$ almost any $\omega$. For further reference, let us call $\Omega_{1}$ the set of $\omega$ 's such that $0 \in \mathcal{C}(\omega), C_{1}(\omega)<\infty$ and $C_{2}(\omega)<\infty$ and observe that $Q_{0}\left(\Omega_{1}\right)=1$.

Define the measure

$$
\mathcal{P}(A)=Q\left[\mathbf{1}_{A}(\omega) n^{\omega}(0) \mathbf{1}_{0 \in \mathcal{C}(\omega)}\right] .
$$

According to the ergodic theorem, for any smooth function $\phi \in C^{\infty}(G)$ and any $u \in L^{1}(\Omega, \mathcal{P})$ we have

$$
\int_{G} \phi(z) u\left(\frac{1}{\varepsilon} z \cdot \omega\right) d \mu_{\omega}^{\varepsilon}(z) \rightarrow\left(\int_{G} \phi(z) d z\right)\left(\int_{\Omega} u\left(\omega^{\prime}\right) d \mathcal{P}\left(\omega^{\prime}\right)\right)
$$

$Q_{0}$.a.s.

We endow $\Omega$ with its natural (product) topology to turn it into a compact space. We will use the notation $C(\Omega)$ for continuous real valued functions defined on $\Omega$. Using standart separability arguments, we see that (2.11) holds simultaneously for any $\phi \in C^{\infty}(G)$ and $u \in C(\Omega)$ on a set of full $Q_{0}$ measure. More precisely, let $\Omega_{2}$ be the set of $\omega$ 's such that $0 \in \mathcal{C}(\omega)$ and, for any functions $\phi \in C^{\infty}(G)$ and $u \in C(\Omega)$ one has:

$$
\int_{G} \phi(z) u\left(\frac{1}{\varepsilon} z \cdot \omega\right) d \mu_{\omega}^{\varepsilon}(z) \rightarrow\left(\int_{G} \phi(z) d z\right)\left(\int_{\Omega} u\left(\omega^{\prime}\right) d \mathcal{P}\left(\omega^{\prime}\right)\right),
$$

and, for any $e \in \mathcal{B}$,

$$
\int_{G} \phi(z) u\left(\frac{1}{\varepsilon} z \cdot \omega\right) G_{b_{0}}\left(\frac{1}{\varepsilon} z \cdot \omega, e\right) d \mu_{\omega}^{\varepsilon}(z) \rightarrow\left(\int_{G} \phi(z) d z\right)\left(\int_{\Omega} u\left(\omega^{\prime}\right) G_{b_{0}}\left(\omega^{\prime}, e\right) d \mathcal{P}\left(\omega^{\prime}\right)\right) .
$$

Then $Q_{0}\left(\Omega_{2}\right)=1$. Finally let $\Omega_{0}=\Omega_{1} \cap \Omega_{2}$.

In the sequel $\alpha$ will denote an element of $\Omega_{0}$. Consider the family of linear functionals

$$
L^{\varepsilon, \alpha}(u, \phi)=\int_{G} \phi(z) \psi^{\varepsilon}(\alpha, z) u\left(\frac{1}{\varepsilon} z \cdot \alpha\right) d \mu_{\alpha}^{\varepsilon}(z) .
$$

Using the Cauchy-Schwartz inequality, we get that

$$
\left(L^{\varepsilon, \alpha}(u, \phi)\right)^{2} \leq \int_{G}\left(\psi^{\varepsilon}(\alpha, z)\right)^{2} d \mu_{\alpha}^{\varepsilon}(z) \int_{G} \phi(z)^{2} u\left(\frac{1}{\varepsilon} z \cdot \alpha\right)^{2} d \mu_{\alpha}^{\varepsilon}(z) .
$$

From (2.10) and (2.12) we deduce that for $\phi \in C^{\infty}(G)$ and $u \in C(\Omega)$

$$
\limsup _{\varepsilon}\left(L^{\varepsilon, \alpha}(u, \phi)\right)^{2} \leq C_{2}(\alpha) \int_{G} \phi(z)^{2} d z \int_{\Omega} u(\omega)^{2} d \mathcal{P}(\omega) .
$$

Therefore, up to extracting a sub-sequence, we can assume that for any smooth $\phi$ and any continuous $u \in C(\Omega)$, $L^{\varepsilon, \alpha}(u, \phi)$ has a limit say $L^{\alpha}(u, \phi)$ where $L^{\alpha}$ is a linear functional satisfying

$$
\left(L^{\alpha}(u, \phi)\right)^{2} \leq C_{2}(\alpha) \int_{G} \phi(z)^{2} d z \int_{\Omega} u(\omega)^{2} d \mathcal{P}(\omega) .
$$

Thus $L^{\alpha}$ can be extended as a continuous linear functional on $L^{2}(\Omega \times G, d \mathcal{P} \times d x)$ and, by Riesz's theorem, there exists a function $v^{\alpha} \in L^{2}(\Omega \times G, d \mathcal{P} \times d x)$ such that

$$
L^{\alpha}(u, \phi)=\int_{G} \phi(z) d z \int_{\Omega} u(\omega) v^{\alpha}(\omega, z) d \mathcal{P}(\omega) .
$$

Let us summarize the preceeding discussion: we have proved that, up to extracting a sub-sequence, for $\phi \in C^{\infty}(G)$ and $u \in C(\Omega)$,

$$
\int_{G} \phi(z) \psi^{\varepsilon}(\alpha, z) u\left(\frac{1}{\varepsilon} z \cdot \alpha\right) d \mu_{\alpha}^{\varepsilon}(z) \rightarrow \int_{G} \phi(z) d z \int_{\Omega} u(\omega) v^{\alpha}(\omega, z) d \mathcal{P}(\omega) .
$$

We will prove the following 
Lemma 2.2 For any $\alpha \in \Omega_{0}, v^{\alpha}(\omega, z)=0$ for Lebesgue almost any $z \in G$ and $\mathcal{P}$ almost any $\omega$.

As a consequence of this Lemma, we have that for $Q_{0}$ almost any $\alpha$, for any function $\phi \in C^{\infty}(G)$,

$$
\int_{G} \phi(z) \psi^{\varepsilon}(\alpha, z) d \mu_{\alpha}^{\varepsilon}(z) \rightarrow 0 .
$$

Since we also have uniform bounds on the $L^{2}$ norm of $\psi^{\varepsilon}$, see (2.10), we deduce that, for any rectangle $A \subset G$,

$$
\int_{A} \psi^{\varepsilon}(\alpha, z) d \mu_{\alpha}^{\varepsilon}(z) \rightarrow 0
$$

We conclude that, for any rectangle $A \subset[-1,1]^{d}, Q_{0}$.a.s.

$$
\varepsilon^{d} \sum_{x \in \mathcal{C}(\omega) ; \varepsilon x \in A}\left(\varepsilon \chi(\omega, x)-a_{\varepsilon}\right) \rightarrow 0 .
$$

Remark 2.3 The content of this part of the paper, including the proof of the Lemma in the next section, should be compared with the results of [9]. The convergence in (2.14) is known as 'two-scale convergence'. The only difference between our setting and [9] is the discrete nature of the grid whereas continuous diffusions are considered in [9].

It is also possible to directly apply the results of [9] to justify Lemma (2.2). We refer the interested reader to the first version of the present paper on the Arxiv for details. Here, we prefered to give a more self-contained approach but most of the arguments are mere copies of the proofs in [9] with some minor simplifications due to the fact that, for instance, the Palm measure $\mathcal{P}$ is explicit and absolutely continuous w.r.t. $Q$.

Proof of Lemma (2.2): the proof is in three steps. Throughout the following proof, $\phi$ is always assumed to be in $C_{o}^{\infty}(G)$, the space of smooth functions with compact support in $G$.

Step 1: we check the integration by parts formula:

$$
\int_{G} \phi(z) \nabla^{(\omega) *} u\left(\frac{1}{\varepsilon} z \cdot \alpha\right) d \mu_{\alpha}^{\varepsilon}(z)=-\varepsilon \int_{G} \frac{1}{n^{\alpha}\left(\frac{1}{\varepsilon} z\right)} \sum_{e \in \mathcal{B}} u\left(\frac{1}{\varepsilon} z \cdot \alpha, e\right)\left(\frac{z}{\varepsilon}\right) \cdot \alpha(e) \nabla_{e}^{\varepsilon} \phi(z) d \mu_{\alpha}^{\varepsilon}(z),
$$

where $u$ is any function defined on $\Omega \times \mathcal{B}$ and $\varepsilon$ is small enough (depending on the support of $\phi$ ):

$$
\begin{aligned}
& \int_{G} \phi(z) \nabla^{(\omega) *} u\left(\frac{1}{\varepsilon} z . \alpha\right) d \mu_{\alpha}^{\varepsilon}(z)=\varepsilon^{d} \sum_{x \in \mathcal{C}(\alpha)} \phi(\varepsilon x) \nabla^{(\omega) *} u(x . \alpha) n^{\alpha}(x) \\
= & \varepsilon^{d} \sum_{x \in \mathcal{C}(\alpha)} \phi(\varepsilon x) \sum_{e \in \mathcal{B}} x . \alpha(e)(u(x . \alpha, e)-u(x . e \cdot \omega,-e)) n^{\alpha}(x) .
\end{aligned}
$$

But

$$
\sum_{x \in \mathcal{C}(\alpha)} \phi(\varepsilon x) \sum_{e \in \mathcal{B}} x . \alpha(e) u(x . e . \alpha,-e) n^{\alpha}(x)=\sum_{x^{\prime} \in \mathcal{C}(\alpha)} \sum_{e^{\prime} \in \mathcal{B}} x^{\prime} \cdot \alpha\left(e^{\prime}\right) u\left(x^{\prime} \cdot \alpha, e^{\prime}\right) \phi\left(\varepsilon x^{\prime}+\varepsilon e^{\prime}\right) n^{\alpha}\left(x^{\prime}\right),
$$

with the change of variables $x^{\prime}=x+e$ and $e^{\prime}=-e$. Putting the last two equalities together, one gets (2.16). Observe that boundary terms vanish because $\phi$ has compact support and $\varepsilon$ is small enough.

Step 2: we prove that $v^{\alpha}(\omega, z)$ does not depend on $\omega$ i.e. that $Q_{0}$.a.s.

$$
v^{\alpha}(\omega, z)=\frac{\int v^{\alpha}\left(\omega^{\prime}, z\right) d \mathcal{P}\left(\omega^{\prime}\right)}{\int d \mathcal{P}\left(\omega^{\prime}\right)}=v^{\alpha}(z) .
$$

Indeed, let $u$ be continuous on $\Omega \times \mathcal{B}$ and $\phi \in C_{o}^{\infty}(G)$ and use (2.14) and the integration by parts formula (2.16) to get that

$$
\int_{G} \phi(z) d z \int_{\Omega} v^{\alpha}(\omega, z) \nabla^{(\omega) *} u(\omega) d \mathcal{P}(\omega)
$$




$$
\begin{aligned}
& =\lim _{\varepsilon} \int_{G} \phi(z) \psi^{\varepsilon}(\alpha, z) \nabla^{(\omega) *} u\left(\frac{1}{\varepsilon} z \cdot \alpha\right) d \mu_{\alpha}^{\varepsilon}(z) \\
& =\lim _{\varepsilon}-\varepsilon \int_{G} \frac{1}{n^{\alpha}\left(\frac{1}{\varepsilon} z\right)} \sum_{e \in \mathcal{B}} u\left(\frac{1}{\varepsilon} z \cdot \alpha, e\right)\left(\frac{z}{\varepsilon}\right) \cdot \alpha(e) \nabla_{e}^{\varepsilon}\left(\psi^{\varepsilon}(\alpha, .) \phi\right)(z) d \mu_{\alpha}^{\varepsilon}(z) .
\end{aligned}
$$

Since $u$ is continuous, it is bounded. Note that $\left(\frac{z}{\varepsilon}\right) \cdot \alpha(e) \leq n^{\alpha}\left(\frac{1}{\varepsilon} z\right)$. Besides,

$$
\limsup _{\varepsilon} \int_{G}\left(\frac{z}{\varepsilon}\right) \cdot \alpha(e)\left(\nabla_{e}^{\varepsilon}\left(\psi^{\varepsilon}(\alpha, .) \phi\right)(z)\right)^{2} d \mu_{\alpha}^{\varepsilon}(z) \leq C_{1}(\alpha)\|\phi\|_{\infty}+C_{2}(\alpha)\|\nabla \phi\|_{\infty}<\infty .
$$

We conclude that, as $\varepsilon$ tends to 0 , the expression

$$
\int_{G} \frac{1}{n^{\alpha}\left(\frac{1}{\varepsilon} z\right)} u\left(\frac{1}{\varepsilon} z \cdot \alpha, e\right)\left(\frac{z}{\varepsilon}\right) \cdot \alpha(e) \nabla_{e}^{\varepsilon}\left(\psi^{\varepsilon}(\alpha, .) \phi\right)(z) d \mu_{\alpha}^{\varepsilon}(z)
$$

remains bounded and therefore

$$
\lim _{\varepsilon}-\varepsilon \int_{G} \frac{1}{n^{\alpha}\left(\frac{1}{\varepsilon} z\right)} \sum_{e \in \mathcal{B}} u\left(\frac{1}{\varepsilon} z \cdot \alpha, e\right)\left(\frac{z}{\varepsilon}\right) \cdot \alpha(e) \nabla_{e}^{\varepsilon}\left(\psi^{\varepsilon}(\alpha, .) \phi\right)(z) d \mu_{\alpha}^{\varepsilon}(z)=0
$$

and

$$
\int_{G} \phi(z) d z \int_{\Omega} v^{\alpha}(\omega, z) \nabla^{(\omega) *} u(\omega) d \mathcal{P}(\omega)=0
$$

By (2.3) we also have

$$
\begin{aligned}
& \int_{\Omega} v^{\alpha}(\omega, z) \nabla^{(\omega) *} u(\omega) d \mathcal{P}(\omega)=\int_{\Omega} v^{\alpha}(\omega, z) \nabla^{(\omega) *} u(\omega) n^{\omega}(0) \mathbf{1}_{0 \in \mathcal{C}(\omega)} d Q(\omega) \\
= & -\int u \nabla^{(\omega)} v^{\alpha}(., z) d M .
\end{aligned}
$$

Thus we have proved that

$$
\int_{G} \phi(z) d z \int u \nabla^{(\omega)} v^{\alpha}(., z) d M=0
$$

for any $\phi \in C_{o}^{\infty}(G)$ and continuous $u$. We deduce that $Q_{0}$.a.s., for any $b \in \mathcal{B}$ such that $\omega(b)=1$ and for Lebesgue almost any $z$, then $v^{\alpha}(b . \omega, z)=v^{\alpha}(\omega, z)$. Integrating this equality on a path between 0 and $x \in \mathcal{C}_{0}(\omega)$, we then get that $Q_{0}$.a.s. for any $x \in \mathcal{C}(\omega)$ and for Lebesgue almost any $z$, then $v^{\alpha}(\omega, z)=v^{\alpha}(x . \omega, z)$. Therefore, since $\mu_{\omega}^{\varepsilon}$ only charges $\mathcal{C}(\omega)$, the ergodic theorem yields:

$$
v^{\alpha}(\omega, z)=\frac{\int_{G} v^{\alpha}\left(\frac{1}{\varepsilon} z^{\prime} \cdot \omega, z\right) d \mu_{\omega}^{\varepsilon}\left(z^{\prime}\right)}{\int_{G} d \mu_{\omega}^{\varepsilon}\left(z^{\prime}\right)} \rightarrow \frac{\int v^{\alpha}\left(\omega^{\prime}, z\right) d \mathcal{P}\left(\omega^{\prime}\right)}{\int d \mathcal{P}\left(\omega^{\prime}\right)}=v^{\alpha}(z),
$$

$Q_{0}$.a.s in $\omega$ and for Lebesgue almost any $z \in G$.

Step 3: we now prove that $v^{\alpha}(z)$ does not depend on $z$. To this end, we first prove that for any smooth $\phi \in C_{o}^{\infty}(G)$ and any continuous $u \in L_{\text {sol }}^{2}$ we have

$$
\sum_{e \in \mathcal{B}}\left(\int_{G} d z v^{\alpha}(z) \nabla \phi(z) \cdot e\right)\left(\int_{\Omega} \tilde{u}(\omega, e) d \mathcal{P}(\omega)\right)=0
$$

where $\tilde{u}(\omega, e)=\frac{\omega(e)}{n^{\omega}(0)} u(\omega, e)$.

We have:

$$
\left(\int_{G} d z v^{\alpha}(z) \nabla \phi(z) \cdot e\right)\left(\int_{\Omega} \tilde{u}(\omega, e) d \mathcal{P}(\omega)\right)
$$




$$
\begin{aligned}
& =\lim _{\varepsilon} \int_{G}(\nabla \phi(z) \cdot e) \psi^{\varepsilon}(\alpha, z) \tilde{u}\left(\frac{1}{\varepsilon} z . \alpha, e\right) d \mu_{\alpha}^{\varepsilon}(z)=\lim _{\varepsilon} \int_{G} \nabla_{e}^{\varepsilon} \phi(z) \psi^{\varepsilon}(\alpha, z) \tilde{u}\left(\frac{1}{\varepsilon} z . \alpha, e\right) d \mu_{\alpha}^{\varepsilon}(z) \\
& =\lim _{\varepsilon} \int_{G} \nabla_{e}^{\varepsilon}\left(\phi \psi^{\varepsilon}(\alpha, .)\right)(z) \tilde{u}\left(\frac{1}{\varepsilon} z . \alpha, e\right) d \mu_{\alpha}^{\varepsilon}(z)-\lim _{\varepsilon} \int_{G} \phi(z) \nabla_{e}^{\varepsilon}\left(\psi^{\varepsilon}(\alpha, .)\right)(z) \tilde{u}\left(\frac{1}{\varepsilon} z . \alpha, e\right) d \mu_{\alpha}^{\varepsilon}(z),
\end{aligned}
$$

where we used (2.14) in the first equality and the regularity of $\phi$ for the second and third equalities. Using integration by parts and the definition of $\tilde{u}$, we get

$$
\begin{aligned}
& \sum_{e \in \mathcal{B}} \int_{G} \nabla_{e}^{\varepsilon}\left(\phi \psi^{\varepsilon}(\alpha, .)\right)(z) \tilde{u}\left(\frac{1}{\varepsilon} z \cdot \alpha, e\right) d \mu_{\alpha}^{\varepsilon}(z) \\
= & \sum_{e \in \mathcal{B}} \int_{G} \nabla_{e}^{\varepsilon}\left(\phi \psi^{\varepsilon}(\alpha, .)\right)(z) \frac{\left(\frac{z}{\varepsilon}\right) \cdot \alpha(e)}{n^{\alpha}\left(\frac{1}{\varepsilon} z\right)} u\left(\frac{1}{\varepsilon} z \cdot \alpha, e\right) d \mu_{\alpha}^{\varepsilon}(z) \\
= & -\frac{1}{\varepsilon} \int_{G} \phi(z) \psi^{\varepsilon}(\alpha, z) \nabla^{(\omega) *} u\left(\frac{1}{\varepsilon} z \cdot \alpha\right) d \mu_{\alpha}^{\varepsilon}(z)=0,
\end{aligned}
$$

since $u \in L_{\text {sol }}^{2}$ and therefore $\nabla^{(\omega) *} u=0$.

We now turn to the second term. Keep in mind that $\nabla_{e}^{\varepsilon}\left(\psi^{\varepsilon}(\alpha,).\right)(z)=G_{b_{0}}\left(\frac{1}{\varepsilon} z . \alpha, e\right)$. Thus, as an application of $(2.13)$,

$$
\lim _{\varepsilon} \int_{G} \phi(z) \nabla_{e}^{\varepsilon}\left(\psi^{\varepsilon}(\alpha, .)\right)(z) \tilde{u}\left(\frac{1}{\varepsilon} z . \alpha, e\right) d \mu_{\alpha}^{\varepsilon}(z)=\left(\int_{G} \phi(z) d z\right)\left(\int_{\Omega} G_{b_{0}}(\omega, e) \tilde{u}(\omega, e) d \mathcal{P}(\omega)\right) .
$$

Replacing $\tilde{u}$ and $\mathcal{P}$ by their definition, we also have

$$
\begin{aligned}
& \sum_{e \in \mathcal{B}} \int_{\Omega} G_{b_{0}}(\omega, e) \tilde{u}(\omega, e) d \mathcal{P}(\omega)=\sum_{e \in \mathcal{B}} \int_{\Omega} G_{b_{0}}(\omega, e) \omega(e) u(\omega, e) \mathbf{1}_{0 \in \mathcal{C}(\omega)} d Q(\omega) \\
= & \int G_{b_{0}} u d M=0
\end{aligned}
$$

since $G_{b_{0}} \in L_{\text {pot }}^{2}$ and $u \in L_{\text {sol }}^{2}$. We conclude that (2.18) holds.

(2.18) was proved for any continuous $u \in L_{\text {sol }}^{2}$. By density it also holds for any $u \in L_{\text {sol }}^{2}$.

It remains to check the following fact: for any direction $e \in \mathcal{B}$, there exists $u \in L_{\text {sol }}^{2}$ such that $\int_{\Omega} \tilde{u}(\omega, e) d \mathcal{P}(\omega) \neq 0$. Indeed, first note that

$$
\int_{\Omega} \tilde{u}(\omega, e) d \mathcal{P}(\omega)=\int_{0 \in \mathcal{C}(\omega)} \omega(e) u(\omega, e) d Q(\omega) .
$$

Define the random field $\tilde{e}$ by $\tilde{e}(\omega, b)=\mathbf{1}_{b=e}$. (e is kept fixed.) Let $G$ be the orthogonal projection of $-\tilde{e}$ on $L_{p o t}^{2}$ and let $u=G+\tilde{e} \in L_{\text {sol }}^{2}$. We write that $u$ and $u-\tilde{e}=G$ are orthogonal:

$$
\int_{0 \in \mathcal{C}(\omega)} \omega(e) u(\omega, e) d Q(\omega)=\int u \tilde{e} d M=\int u^{2} d M \neq 0
$$

because $\tilde{u} \notin L_{\text {sol }}^{2}$.

Thus we can deduce from (2.18) that $\int_{G} d z v^{\alpha}(z) \nabla \phi(z) \cdot e=0$ for any smooth $\phi$ and any direction $e$. Therefore $v^{\alpha}$ is Lebesgue almost surely constant.

Conclusion of the proof of Lemma (2.2): since $\psi^{\varepsilon}$ has vanishing mean on $G$ - Remember this is the way we chose $a_{\varepsilon}$ - then $v^{\alpha}$ also has vanishing mean in $G$. And since, by steps 2 and 3 , $v^{\alpha}$ is almost surely constant, we must have that $Q_{0}$.a.s. and for Lebesgue almost any $z, v^{\alpha}(\omega, z)=0$.

\section{Scaling and strong $L^{2}$ convergence of $\chi$ :}

To conclude the proof of the Theorem, we still have to prove the strong $L^{2}$ convergence in (2.6). It will be a consequence of the weak convergence (2.15) and of a scaling argument.

We choose a parameter $\delta>0$. We chop the box $[-1,1]^{d}$ into smaller boxes of side length of order $\delta$ : for $z \in \delta \mathbb{Z}^{d}$ s.t. $|z| \leq 1$, let $B_{z}$ (resp. $C_{z}$ ) be the box of center $z$ and side length $M \delta$ (resp. side length $\delta$ ). 
$M$ is a constant whose value will be chosen later. For $\varepsilon>0$, we use the notation $B_{z}(\varepsilon)=\left(\frac{1}{\varepsilon} B_{z}\right) \cap \mathbb{Z}^{d}$ and $C_{z}(\varepsilon)=\left(\frac{1}{\varepsilon} C_{z}\right) \cap \mathbb{Z}^{d}$

The following version of the Poincaré inequality is proved in [1], see Definition 1.7, Theorem 2.18, Lemma 2.13 and Proposition 2.17: there exist constants $M>1$ and $\beta$ such that $Q_{0}$.a.s. for any $\delta>0$, for small enough $\varepsilon$, for any $z \in \delta \mathbb{Z}^{d}$ s.t. $|z| \leq 1$ and for any function $u: \mathbb{Z}^{d} \rightarrow \mathbb{R}$ one has

$$
\frac{1}{\# C_{z}(\varepsilon)} \sum_{x, y \in \mathcal{C}(\omega) \cap C_{z}(\varepsilon)}(u(x)-u(y))^{2} \leq \beta \delta^{2} \varepsilon^{-2} \sum_{x \sim y \in \mathcal{C}(\omega) \cap B_{z}(\varepsilon)} \omega(x, y)(u(x)-u(y))^{2} .
$$

We use this inequality for the function $\varepsilon \chi$, to get that

$$
\frac{1}{\# C_{z}(\varepsilon)} \sum_{x, y \in \mathcal{C}(\omega) \cap C_{z}(\varepsilon)}(\varepsilon \chi(\omega, x)-\varepsilon \chi(\omega, y))^{2} \leq \beta \delta^{2} \sum_{x \in \mathcal{C}(\omega) \cap B_{z}(\varepsilon)} \sum_{b \in \mathcal{B}} \sum_{e \in \mathcal{B}} \omega(x, y)\left(G_{b}(\omega, e)\right)^{2} .
$$

Denoting with $a_{\varepsilon}(z)$ the mean value of $\varepsilon \chi(\omega,$.$) on the set \mathcal{C}(\omega) \cap C_{z}(\varepsilon)$, we get that for all $z$,

$$
\sum_{x \in \mathcal{C}(\omega) \cap C_{z}(\varepsilon)}\left(\varepsilon \chi(\omega, x)-a_{\varepsilon}(z)\right)^{2} \leq \beta \delta^{2} \sum_{x \in \mathcal{C}(\omega) \cap B_{z}(\varepsilon)} \sum_{b \in \mathcal{B}} \sum_{e \in \mathcal{B}} \omega(x, y)\left(G_{b}(\omega, e)\right)^{2},
$$

and summing over all values of $z$,

$$
\sum_{z} \sum_{x \in \mathcal{C}(\omega) \cap C_{z}(\varepsilon)}\left(\varepsilon \chi(\omega, x)-a_{\varepsilon}(z)\right)^{2} \leq \beta \delta^{2} \sum_{x \in \mathcal{C}(\omega) ;|x| \leq 1 / \varepsilon} \sum_{\varepsilon \in \mathcal{B}} \sum_{e \in \mathcal{B}} \omega(x, y)\left(G_{b}(\omega, e)\right)^{2} .
$$

(Remember that the value of $\beta$ is allowed to change from line to line.) Multiplying by $\varepsilon^{d}$ and applying the spatial ergodic theorem as before, we get that

$$
\limsup _{\varepsilon} \sum_{z} \varepsilon^{d} \sum_{x \in \mathcal{C}(\omega) \cap C_{z}(\varepsilon)}\left(\varepsilon \chi(\omega, x)-a_{\varepsilon}(z)\right)^{2} \leq \beta \delta^{2} \sum_{b \in \mathcal{B}} \int\left(G_{b}\right)^{2} d M .
$$

On the other hand, it follows from (2.15) that, for any $z, a_{\varepsilon}(z)-a_{\varepsilon}$ converges to 0 . Therefore we must also have

$$
\limsup _{\varepsilon} \varepsilon^{d} \sum_{z} \sum_{x \in \mathcal{C}(\omega) \cap C_{z}(\varepsilon)}\left(\varepsilon \chi(\omega, x)-a_{\varepsilon}\right)^{2} \leq \beta \delta^{2} \sum_{b \in \mathcal{B}} \int\left(G_{b}\right)^{2} d M
$$

and

$$
\limsup _{\varepsilon} \varepsilon^{d} \sum_{x \in \mathcal{C}(\omega) ;|x| \leq 1 / \varepsilon}\left(\varepsilon \chi(\omega, x)-a_{\varepsilon}\right)^{2} \leq \beta \delta^{2} \sum_{b \in \mathcal{B}} \int\left(G_{b}\right)^{2} d M
$$

and, since this holds for any $\delta>0$, we deduce that

$$
\varepsilon^{d} \sum_{x \in \mathcal{C}(\omega) ;|x| \leq 1 / \varepsilon}\left(\varepsilon \chi(\omega, x)-a_{\varepsilon}\right)^{2} \rightarrow 0
$$

$Q_{0}$.a.s. 


\section{References}

[1] Barlow, M.T. (2004)

Random walks on supercritical percolation clusters

Ann. Probab. 32, 3024-3084.

[2] Berger, N., Biskup, M. (2005)

Quenched invariance principle for simple random walk on percolation clusters. Preprint.

http://front.math.ucdavis.edu/math.PR/0503576.

[3] De Masi, A., Ferrari, P., Goldstein, S., Wick, W.D. (1989)

An invariance principle for reversible Markov processes. Applications to random motions in random environments

Journ. Stat. Phys. 55 (3/4), 787-855.

[4] Ethier, S.N., Kurtz, T.G. (1986)

Markov processes

John Wiley, New York.

[5] Grimmett, G. (1999)

Percolation

Springer-Verlag, Berlin (Second edition).

[6] Grimmett, G., Marstrand, J. (1990)

The supercritical phase of percolation is well behaved

Proc. Royal Society (London) Ser. A. 4306, 429-457.

[7] Helland, I (1982)

Central limit theorems for martingales with dicrete or continuous time

Scand. Journ. Stat. 9, 79-94.

[8] Jikov, V.V., Kozlov, S.M., Oleinik, O.A. (1994)

Homogenization of differential operators and integral functionals.

Springer-Verlag, Berlin.

[9] Jikov, V.V., Piatnitski, A.L. (2005)

Homogenization of random singular structures and measures.

Preprint (in Russian).

[10] Kozlov, S.M. (1985)

The method of averaging and walks in inhomogeneous environments

Russian Math. Surveys 40 (2), 73-145.

[11] Krengel, U (1985)

Ergodic theorems

Walter de Gruyter, Berlin.

[12] Mathieu, P., Remy, E. (2004)

Isoperimetry and heat kernel decay on percolations clusters

Ann. Probab. 32, 100-128.

[13] Sidoravicius, V., Sznitman, A-S. (2004)

Quenched invariance principles for walks on clusters of percolation or among random conductances Prob. Th. Rel. Fields 129, 219-244. 